\title{
Desafíos de las publicaciones científicas en la pandemia por el nuevo coronavirus (SARS-CoV-2)
}

\author{
Dres. Carolina Scasso*, Julio Medina ${ }^{\dagger}$
}

\section{Resumen}

La pandemia por el nuevo coronavirus (SARS-CoV-2) trajo cambios inesperados en la forma en la que se produce y publica el conocimiento científico, pero eventos recientes encendieron el debate sobre la calidad de la información que se está divulgando. En el contexto de la pandemia, la comunidad científica ha respondido aumentando en forma exponencial sus investigaciones y haciéndolas rápidamente accesibles. Esto si bien tiene el objetivo loable de generar avances en el conocimiento del SARS-CoV-2 (COVID-19), trae ciertos riesgos aparejados.

En este artículo de opinión abordamos varios tópicos: 1) el acceso libre, a fin de compartir la literatura científica relevante de forma abierta y rápida; 2) el impacto de los repositorios más conocidos de preprints, como medRxiv, aRxiv, bioRxiv y ChemRxiv; 3) la utilización de inteligencia artificial aplicada al análisis de datos, por ejemplo COVID-19 Open Research Dataset (CORD-19) o SciSight; 4) las estrategias para mantener el rigor científico, ya sea a través de una curaduría abierta por la comunidad científica o a través de Outbreak Science Rapid PREreview de la Wellcome Trust, o el 2019 Novel Coronavirus Research Compendium (NCRC); 5) finalmente, abordamos los dilemas de la revisión por pares que busca mejorar la transparencia de este proceso. Varios autores impulsan que el arbitraje abierto sea la norma, esto quiere decir que se publique el manuscrito original junto con su revisión, incluyendo o no el nombre de los revisores.

Se podrá apreciar como no todo lo publicado mantiene los estándares de calidad requeridos y la velocidad conspira contra la buena ciencia. Debemos considerar lo publicado en preprint como datos preliminares, y aun frente a artículos provenientes de revistas auditadas, sería razonable mantener cierto grado de escepticismo. Es imprescindible la revisión sistemática de los estudios científicos de la forma tradicional o con las nuevas herramientas que se han desarrollado en esta pandemia. Las políticas en salud y los estándares de cuidados deben estar basados en conocimientos sólidos, obtenidos de forma transparente, para que incluso en momentos de incertidumbre todos los actores estemos convencidos en que el camino indicado es el mejor con la información que se dispone por el momento.

Palabras clave: Coronavirus

Publicaciones científicas y técnicas

Difusión de la información
Key words: $\quad$ Coronavirus

Scientific and technical publications

Information dissemination

\footnotetext{
* Posgrado de Neurología.

† Prof. Director de la Cátedra de Enfermedades Infecciosas, Facultad de Medicina, Universidad de la República. Uruguay.

Correo electrónico: clinfec@fmed.edu.uy
} 


\section{Introducción}

La pandemia por el nuevo coronavirus (SARS-CoV-2) trajo cambios inesperados en la forma en la que se produce y publica el conocimiento científico, pero eventos recientes encendieron el debate sobre la calidad de la información que se está divulgando ${ }^{(1)}$. La imperiosa necesidad de contar en forma rápida con evidencia sólida que guíe las decisiones de los responsables de las políticas de salud, de los equipos de salud en primera línea y de la comunidad científica, ha aumentado la presión sobre los investigadores a la hora de producir y divulgar material científico, y, por contrapartida, ha cambiado los contrapesos y controles habituales de esta actividad. La Red de Reproducibilidad del Reino Unido (UKRN, por su sigla en inglés) fue creada en 2019 para mejorar la calidad de la investigación, su presidente, Munafò M, manifestó en cuanto a esto: "Se necesitan urgentemente datos y conocimiento, pero la información falsa es peor que la ausencia de información. Debemos asegurarnos que la velocidad en la generación del conocimiento no sea a expensas de su calidad"(2). Para obtener conocimiento de calidad, que forme evidencia científica, se requiere transparencia, rigurosidad, controles y que los resultados puedan ser replicados (una forma de testear que las observaciones originales sean robustas), esto no es lineal y necesariamente lleva tiempo. Se admite que en esta situación excepcional se pueda aceptar un estándar diferente al habitual; sin embargo, esto no cambia la debida diligencia exigida a los científicos ${ }^{(3)}$.

La publicación reciente de artículos con serios problemas metodológicos por dos de las revistas de divulgación científica médica más prestigiosas, trajo al tapete la discusión acerca de cómo se están difundiendo y la calidad de los contenidos de los estudios científicos en estos días. La anécdota en sí es sencilla. Un artículo publicado por The Lancet, el 22 de mayo, reportó un aumento en la mortalidad por arritmias de pacientes que utilizaban cloroquina e hidroxicloroquina ${ }^{(4)}$, lo cual despertó la suspicacia de científicos en todo el mundo. Se trataba de un estudio retrospectivo, de tipo observacional, que utilizó una supuesta base de datos de 96.000 pacientes con diagnóstico de COVID-19 ingresados a 671 hospitales alrededor del mundo. Diferentes investigadores alertaron sobre incongruencias en los números de casos y decesos ${ }^{(5)}$, diferencias llamativas con los resultados que estaban obteniendo en sus propios estudios diseñados ad hoc para estas drogas, e incluso se cuestionó la metodología empleada. A todo esto, se sumó una investigación periodística de The Guardian ${ }^{(6)}$ que arrojó dudas sobre la idoneidad de Surgisphere, la novel y pequeña empresa de análisis de datos en salud que proveyó la base utilizada por este y por otro estudio divulgado en la New England Journal of Medicine (NEJM) acerca de la inocuidad de los inhibidores de la enzima conversora de angiotensina (IECA) y los antagonistas del receptor de angiotensina (ARA2) para los pacientes con COVID$19^{(7)}$. Tanto The Lancet $^{(8)}$ como la $N E J M^{(9)}$ publicaron notas, el pasado 2 de junio, expresando su preocupación por la calidad de la información vertida en estos estudios $\mathrm{y}$ anunciaron auditorías independientes, pero finalmente terminaron por retirar los artículos en forma definitiva al no poder acceder a la base de datos empleada ${ }^{(10,11)}$.

\section{Acceso libre}

Diferentes organizaciones, como Wellcome Trust ${ }^{(12)}$ y la Organización Mundial de la Salud (OMS), apelaron a la colaboración internacional de investigadores, academia, sociedades científicas y editoriales a fin de compartir la literatura científica relevante de forma abierta y rápida, para potenciar así la investigación que busca, entre otras cosas, apoyar el desarrollo de vacunas eficientes y seguras, y de intervenciones farmacéuticas más efectivas. Las editoriales han contribuido permitiendo el acceso libre a parte de sus contenidos relacionados con el SARS-CoV-2 y disminuyendo los plazos habituales de publicación, los cuales serían inaceptables en el momento actual ${ }^{(13)}$. A pesar de esto, $20 \%$ de las investigaciones relacionadas a SARS-CoV-2 publicadas por las revistas académicas serían de acceso restringido para suscriptores ${ }^{(14)}$. Algunos se cuestionan si esta aceleración de los procesos no trajo aparejada una disminución en los controles imprescindibles. Hay que tener en cuenta que, además, en los primeros meses del año aumentó exponencialmente la cantidad de manuscritos relacionados con COVID-19 enviados a las revistas científicas y que el tiempo en la que se realiza la revisión por pares ha disminuido, en algunos casos casi a la mitad, de 117 días a 60 en promedio ${ }^{(15)}$. Por otro lado, desde las editoriales se argumenta que está aumentando la cantidad de manuscritos enviados de baja calidad ${ }^{(16)}$. Un gran número de estos contienen solo comentarios o son protocolos, o modelos de proyección de baja calidad. Malcolm Macleod, profesor de Neurología y Neurociencia translacional de la Universidad de Edimburgo, integra un equipo que analiza las publicaciones científicas desde el inicio de la pandemia. De las que lograron categorizar, sólo el $14 \%$ eran trabajos de investigación primaria, dentro de éstos, tan sólo el 27\% había sido revisado por pares y el $72 \%$ se trataba de trabajos de tipo observacional ${ }^{(3)}$.

\section{Prepints y repositorios}

Por otro lado, se popularizaron las plataformas que divulgan en forma abierta manuscritos preprint. Si bien eran utilizadas por la física y las matemáticas desde la década de 1990, su uso para las ciencias médicas no se 
extendió hasta el momento actual. Los dos principales servidores vinculados a la difusión de material científico médico son bioRxiv ${ }^{(17)}$, creado en 2013, que se encuentra alojado en el laboratorio Cold Spring Harbor (CSHL, por su sigla en inglés) de Nueva York y su hermano más joven medRxiv ${ }^{(18)}$ (también propiedad del CSHL, junto con la British Medical Journal y la Universidad de Yale), fundado en 2019. En estos repositorios se difunden en mucho menor tiempo (dos a cinco días) trabajos de investigación completos, no publicados previamente, pero que no pasan por una revisión por pares ${ }^{(15,16)}$. Es de notar que las revistas auditadas también difunden en sus páginas web artículos bajo el rótulo on-line first y éstos no siempre han sido previamente revisados por pares. Se desprende de un análisis de la literatura relacionada con COVID-19, publicado en bioRxiv, que el $80 \%$ de los manuscritos analizados aparecieron en revistas auditadas, algunos de los cuales habían sido publicados primero como preprints ${ }^{(14)}$.

Hasta la segunda quincena del mes de mayo, en total, habrían aparecido entre 13.700 y 23.000 trabajos sobre SARS-CoV-2 o COVID-19, según las distintas fuentes ${ }^{(14,19)}$. A este ritmo se proyectó que las publicaciones se duplicarían cada 20 días ${ }^{(20)}$. Al menos 4.000 de estas publicaciones se encontraban en los repositorios más conocidos de preprints (medRxiv, aRxiv, bioRxiv y ChemRxiv) ${ }^{(16)}$. El repositorio con mayor número de artículos vinculados al coronavirus-2019 es PMC, seguido de medRxiv ${ }^{(20)}$. El Instituto Europeo de Bioinformática proyectó que se habrían publicado en mayo, en todas las plataformas, en promedio más de 850 estudios por semana, con un pico en la semana del 4 de mayo con más de 930 , de los cuales 458 fueron difundidos por bioRxiv y medRxiv ${ }^{(21)}$.

\section{Inteligencia artificial aplicada al análisis de datos}

Este aluvión de estudios hace que sean inabarcables, lo que motivó a que desde diversas organizaciones se generaran bases de datos para poder indexarlos, acceder a sus contenidos y así poder analizarlos sistemáticamente. Dos ejemplos son la base de datos COVID-19 Open Research Dataset (CORD-19), desarrollada para la Oficina de Políticas de Ciencia y Tecnología de la Casa Blanca por un grupo de expertos que incluye a Google, la Iniciativa Chan Zuckerberg, el Instituto Allen para la inteligencia artificial (IA), entre otros, con los Institutos Nacionales de Salud ${ }^{(14)}$, y la base de datos COVID-19 de la OMS. Para el análisis de estas extensas bases de datos se utilizan herramientas de IA y técnicas de minería de datos. Por ejemplo, la Casa Blanca les encargó a científicos de datos herramientas para analizar las publicaciones contenidas en CORD-19. Con este fin crearon algoritmos para cosechar datos, éstos identifican los artículos con la información relevante, los subdividen en categorías, y generan una página web para cada tema ordenando los resultados obtenidos en cada artículo científico en una tabla. Sin embargo, los algoritmos no siempre extrajeron la información relevante, por lo cual los resultados debieron ser corroborados por voluntarios ${ }^{(14)}$. Otro ejemplo es SciSight ${ }^{(22)}$, desarrollada con el apoyo del Instituto Allen y científicos de la Universidad de Washington, Seattle. Este instrumento de búsqueda de IA, también diseñada para CORD-19, estaría más enfocada en el usuario. No obstante, estas herramientas son poco conocidas por la comunidad científica, a algunos usuarios la interfase les resulta poco amigable, están habituados a otras herramientas de búsqueda y por ende todavía son poco empleadas $^{(14)}$.

\section{Estrategias para mantener el rigor científico}

Si bien la rápida difusión del conocimiento es la principal virtud de las plataformas que incluyen preprints, el no pasar por una estricta revisión por pares conlleva el riesgo de incluir datos y resultados incorrectos o fallas metodológicas que invaliden sus resultados, o sea, de contener "mala ciencia". Si bien, bioRxiv y medRxiv aplican ciertos controles de calidad que tienen como fin evitar plagios, omisiones o que se publiquen contenidos que impliquen riesgos para la bioseguridad o la salud.

Teniendo en cuenta lo anterior han surgido esfuerzos por realizar una curaduría de estas publicaciones. Por un lado, surgió la llamada "revisión de la comunidad", una fluida interrelación entre los actores de la comunidad científica a partir de la cual los errores, aun los más pequeños, las omisiones de datos o de la metodología empleada, son señalados en tiempo real. En general, los autores aceptan estas críticas corrigiendo o incluso retirando los documentos ${ }^{(23)}$. Esta forma novedosa de curaduría se da en gran medida en las redes sociales, por ejemplo, Twitter, y es una de las ventajas de los preprints, que rápidamente pueden ser evaluados por una gran cantidad de investigadores y académicos. También surgieron otras más formales, como el Outbreak Science Rapid PREreview de la Wellcome Trust, o el 2019 Novel Coronavirus Research Compendium (NCRC) de la Universidad John Hopkins. En otro intento de abarcar este problema, algunas editoriales comerciales y académicas realizaron un llamado a interesados para formar listas de revisores para una evaluación rápida de los manuscritos $^{(16)}$. Una de estas iniciativas es Review Commons, una plataforma creada por algunas editoriales y revistas como PLOS, eLife y UK Royal Society, que ofrece, según define su sitio web, "una revisión por pares para las ciencias biológicas de alta calidad, independiente de las revistas"(24). En este sitio los autores presentan su manuscrito, que es seleccionado y enviado a 
revisores, el análisis por ellos realizado, junto con el manuscrito, y la respuesta del autor, es entonces publicada en bioRxiv, y presentada a las revistas asociadas a la plataforma de manera que puede terminar como un trabajo publicado.

Otros investigadores, como Lakens D, de la Universidad de Eindhoven, Holanda, postula que la revisión debería de surgir a la par de la investigación. Los investigadores deberían reclutar un "equipo rojo", un grupo de científicos no relacionado directamente con el proyecto, capaces de analizarlo y criticar sus fallas en cada momento. El nivel de comunicación que puede lograrse entre ambos equipos redunda en manuscritos de mejor calidad y con mayores oportunidades de ser publicados ${ }^{(25)}$.

En esa misma línea se ubica Munafò $\mathrm{M}^{(2)}$, para quien la transparencia en el desarrollo de una investigación es la clave para mejorar el resultado final y evitar errores. "Al poner a disposición de los investigadores los protocolos, materiales, datos, planes de análisis, se podrá escudriñar el trabajo de cerca mientras este se desarrolla y si hay errores estos serán detectados y corregidos más rápidamente", afirma.

Chambers C, en la misma línea, es defensor del modelo de reporte registrado (RR) como otra forma de evitar el error en las investigaciones. Este ya es utilizado por algunas revistas auditadas e implica que la revisión por pares se realiza antes y durante la investigación. Los autores diseñan su trabajo de investigación, en un manuscrito lo fundamentan, generan las hipótesis pertinentes, explican con qué metodología las comprobarán y envían esto para la corrección por pares. Si este manuscrito inicial es aprobado, los autores tienen la seguridad de que su estudio será publicado cuando esté concluido. Inicialmente este manuscrito es publicado en un repositorio como Open Science Framework. En una segunda etapa, luego que se realiza el trabajo de campo y se llega a los resultados, el trabajo completo es nuevamente enviado a revisión y finalmente publicado. Los defensores de este modelo apuntan a que es transparente, eficiente, que se lo puede estandarizar. Los detractores afirman que es rígido y que no es aplicable a todos los tipos de investigaciones ${ }^{(26)}$.

\section{Los dilemas de la revisión por pares}

La revisión por pares es la estrategia universalmente aceptada que asegura un mínimo estándar de calidad en el trabajo auditado y que los resultados difundidos son confiables y relevantes para el avance del conocimiento científico. Se trata del análisis independiente de al menos dos expertos en el tema cuyo objetivo es encontrar errores y mejorar la calidad de la investigación. Este es un proceso indispensable, no siempre remunerado, y por el cual los revisores, en general, no obtienen reco- nocimiento. Pero no es infalible -dado que buenos trabajos pueden recibir críticas injustificadas y otros con falencias pasar la revisión sin problemas- y ha sido tildado por algunos de poco transparente, se le cuestiona su efectividad, calidad y lentitud.

Para darle transparencia a este proceso, varios autores impulsan que el arbitraje abierto sea la norma, esto quiere decir que se publique el manuscrito original junto con su revisión, incluyendo o no el nombre de los revisores. De esta manera, además de arrojar luz sobre la auditoría realizada, se evitan las revisiones fraudulentas, el robo de las investigaciones por parte de los revisores, o que presionen a los autores a que citen sus propios estudios para obtener una buena revisión ${ }^{(27)}$. Ya existen publicaciones que adoptaron esta práctica de forma habitual, por ejemplo BMC Medicine y F1000 Research. También se crearon servidores, como Publons, que buscan revalorizar el trabajo de los revisores y darles el reconocimiento que su labor merece. En este sitio los académicos pueden publicar sus arbitrajes o listar las revistas y organizaciones académicas para las cuales trabajan.

Otra iniciativa en este mismo sentido es la Plataforma de Políticas Editoriales Responsables (PREP), fundada por la Organización Holandesa para la Investigación y el Desarrollo en Salud, y el Centro de Estudios de Ciencia y Tecnología de la Universidad de Leiden. Su cometido es evaluar de forma transparente los procedimientos de revisión por pares de 387 revistas científi$\operatorname{cas}^{(28)}$.

\section{Conclusiones}

En el contexto de la pandemia, la comunidad científica ha respondido aumentando en forma exponencial sus investigaciones y haciéndolas rápidamente accesibles. Esto si bien tiene el objetivo loable de generar avances en el conocimiento del SARS-CoV-2 (COVID-19), trae ciertos riesgos aparejados. Como fue dicho, no todo lo publicado mantiene los estándares de calidad requeridos y la velocidad conspira contra la buena ciencia. Debemos considerar lo publicado en preprint como datos preliminares y aun frente a artículos provenientes de revistas auditadas sería razonable mantener cierto grado de escepticismo. Recordar que gran parte de trabajos en prensa son de tipo observacional e incluso los que involucran un alto número de casos aportan un nivel de evidencia bajo. Es imprescindible la revisión sistemática de los estudios científicos de la forma tradicional o con las nuevas herramientas que se han desarrollado en esta pandemia. Porque las políticas en salud y decisiones a nivel individual de las personas deben estar basadas en conocimientos sólidos, obtenidos de forma transparente, para que aun en momentos de incertidumbre todos 
los actores estemos convencidos en que el camino indicado es el mejor con la información que se dispone por el momento.

Citando a Munafò M, "el conocimiento evolucionará y ningún estudio o hallazgo será definitivo. Entender la COVID-19 es un esfuerzo colectivo".

\section{Summary}

The COVID-19 (SARS-CoV-2) pandemic has resulted in unexpected changes in the way in which scientific knowledge is produced and published, although recent events sparked the debate on the quality of the information that is being disseminated. Within the context of the pandemic, the scientific community has responded by exponentially increasing research and making findings rapidly known. Despite this being a worthy action towards making progress in the knowledge about the COVID-19, it also entails certain risks.

This opinion article addresses several topics: 1) free access with the purpose of sharing relevant scientific literature in an open and fast mode; 2) the impact of the most well-known preprint information repositories such as medRxiv, aRxiv, bioRxiv y ChemRxiv; 3) the use of artificial intelligence applied to data analysis, for example COVID-19 Open Research Dataset (CORD-19) or SciSight ; 4) the strategies to maintain scientific rigour, be it through an open guardianship by the scientific community or by means of the Outbreak Science Rapid PREreview of the Wellcome Trust or the 2019 Novel Coronavirus Research Compendium (NCRC); 5) Last, it addresses the dilemmas of peer review that seeks to improve the process's transparency. Thus, several authors encourage open referral to be the norm, what requires the original text to be published along with its review, with or without the inclusion of the reviewers' names.

It may be appreciated that not all that is published observes the required quality standards and that speed undermines good science. We must consider what is published in preprint as preliminary data, and it would be advisable to hold a certain degree of skepticism even in the case of articles from audited journals. The systematic review of scientific research is of the essence, be it the traditional one or aided by the new tools that have been developed in this pandemic. The health policies and standards of care must be based on solid knowledge, obtained in a transparent way, so that even in times of uncertainty, all the actors are positive that the suggested course of action is the best one in the light of the information available at the time.

\section{Resumo}

A pandemia pelo novo coronavirus (SARS-CoV-2) causou mudanças inesperadas sobre a forma em que se pro- duz e se publica o conhecimento científico. Eventos recentes acenderam o debate sobre a qualidade da informação que está sendo divulgada. No contexto da pandemia, a comunidade científica respondeu aumentando exponencialmente o número de pesquisas e a rapidez em que estavam acessíveis. Embora tivessem o objetivo louvável de gerar avanços no conhecimento do SARS-CoV-2 (COVID-19) esta situação trouxe alguns riscos.

Neste artículo de opinião abordamos vários tópicos como : 1) o livre acesso para compartilhar a literatura científica relevante de forma aberta e rápida; 2) o impacto dos repositórios más conhecidos de preprints como medRxiv, aRxiv, bioRxiv e ChemRxiv; 3) a utilização de inteligência artificial aplicada a análise de dados, por exemplo, COVID-19 Open ResearchDataset (CORD-19) ou SciSight; 4) as estratégias para manter o rigor científico - seja através de uma curadoria aberta pela comunidade científica ou através de OutbreakScience Rapid PREreview do Wellcome Trust, ou o 2019 Novel Coronavirus Research Compendium (NCRC); 5) Finalmente, abordamos os dilemas da revisão por pares que busca melhorar a transparência deste processo. Assim, vários autores impulsam que a arbitragem aberta seja a norma, quer dizer - que se publique o manuscrito original junto com sua revisão, incluindo ou não o nome dos revisores.

Pode-se observar como não tudo o que foi publicado manteve os padrões de qualidade requeridos e como a velocidade conspira contra a boa ciência. Devemos considerar o que é publicado como preprint como dados preliminares e, que mesmo frente aos artigos publicados em revistas auditadas seria razoável manter certo grau de ceticismo. A revisão sistemática dos estudos científicos é imprescindível, seja feita da forma tradicional ou utilizando as novas ferramentas desenvolvidas durante esta pandemia. As políticas de saúde e os padrões de cuidados devem estar baseados em conhecimentos sólidos, obtidos de forma transparente, para que mesmo em momentos de incerteza, todos os autores estejamos convencidos de que o caminho indicado é o melhor com a informação disponível no momento.

\section{Bibliografía}

1. Iacobucci G. Covid-19: validity of key studies in doubt after leading journals issue expressions of concern. BMJ 2020; 369:m2224. doi: 10.1136/bmj.m2224.

2. Munafo M. What you need to know about how coronavirus is changing science. The Conversation 2020. Disponible en: https://theconversation.com/what-you-need-to-knowabout-how-coronavirus-is-changing-science-137641[Consulta: 4 de junio de 2020].

3. Armstrong S. Research on covid-19 is suffering "imperfect incentives at every stage". BMJ 2020; 369:m2045. doi: 10.1136/bmj.m2045. 
4. Mehra MR, Desai SS, Ruschitzka F, Patel AN. Hydroxychloroquine or chloroquine with or without a macrolide for treatment of COVID-19: a multinational registry analysis. Lancet 2020; S0140-6736(20):31180-6. doi:10.1016/S0140-6736(20)31180-6.

5. Davey M. Questions raised over hydroxychloroquine study which caused WHO to halt trials for Covid-19. The Guardian 2020. Disponible en: https://www.theguardian.com /science/2020/may/28/questions-raised-over-hydroxychloroquine-study-which-caused-who-to-halt-trials-for-covid-19 [Consulta: 4 de junio de 2020].

6. Davey M, Kirchgaessner S, Boseley S. Surgisphere: governments and WHO changed Covid-19 policy based on suspect data from tiny US company. The Guardian 2020. Disponible en: https://www.theguardian.com/world/2020/jun/03/ covid-19-surgisphere-who-world-health-organizationhydroxychloroquine [Consulta: 11 de junio de 2020].

7. Mehra MR, Desai SS, Kuy S, Henry TD, Patel AN. Cardiovascular disease, drug therapy, and mortality in Covid-19. N Engl J Med 2020; 382(25):e102. doi: 10.105 6/NEJMoa 2007621.

8. The Lancet Editors. Expression of concern: hydroxychloroquine or chloroquine with or without a macrolide for treatment of COVID-19: a multinational registry analysis. Lancet 2020;395(10240):e102. Disponible en: https://marlinprod.literatumonline.com/pb-assets/Lancet/pdfs/S01406 73620312903.pdf [Consulta: 4 de junio de 2020].

9. Rubin EJ. Expression of concern: Mehra MR et al. Cardiovascular disease, drug therapy, and mortality in Covid-19. N Engl J Med. DOI: 10.1056/NEJMoa2007621. N Engl J Med 2020; 382(25):2464. doi: 10.1056/NEJMe2020822.

10. Mehra MR, Desai SS, Kuy S, Henry TD, Patel AN. Retraction: Cardiovascular disease, drug therapy, and mortality in Covid-19. N Engl J Med 2020. doi: 10.1056/NEJMoa 2007621. [retraction of: N Engl J Med. 2020 Jun 18;382(25): e102]. N Engl J Med. 2020;382(26):2582. doi: 10.1056/ NEJMc2021225.

11. Mehra MR, Desai SS, Ruschitzka F, Patel AN. Retracted: Hydroxychloroquine or chloroquine with or without a macrolide for treatment of COVID-19: a multinational registry analysis. Lancet 2020; S0140-6736(20):31180-6. doi: https://10.1016/S0140-6736(20)31180-6.

12. Carr D. Sharing research data and findings relevant to the novel coronavirus (COVID-19) outbreak. 31 jan. 2020. Disponible en: https://wellcome.ac.uk/coronavirus-covid-19/ open-data [Consulta: 7 de junio de 2020].

13. Kiley R. Open access: how COVID-19 will change the way research findings are shared. 21 may. 2020. Disponible en: https://wellcome.ac.uk/news/open-access-how-covid-19will-change-way-research-findings-are-shared. [Consulta: 4 de junio de 2020].

14. Brainard J. Scientists are drowning in COVID-19 papers. Can new tools keep them afloat? Science May 132020. doi:10.1126/science.abc7839.

15. Callaway E. Will the pandemic permanently alter scientific publishing? Nature 2020; 582(7811):167-8. doi: 10.1038/d41586-020-01520-4.

16. Kwon D. How preprint servers are blocking bad coronavirus research. Nature 2020; 581(7807):130-1. doi: 10.1038/d41586-020-01394-6.

17. BioRvxiv. Disponible en: https://www.biorxiv.org [Consulta: 10 de junio de 2020].

18. MedXriv. Disponible en: https://www.medrxiv.org [Consulta: 10 de junio de 2020].

19. Wigginton NS, Cunningham RM, Katz RH, Lidstrom ME, Moler KA, Wirtz D, et al. Moving academic research forward during COVID-19. Science 2020; 368(6496):1190-2. doi: 10.1126/science.abc5599.

20. Torres-Salinas D, Robinson-Garcia N, Castillo-Valdivieso P. Open access and altmetrics in the pandemic age: forescast analysis on COVID-19 literature. BioRxiv 2020.04.23.057307. doi: https://doi.org/10.1101/2020.04.23.057307.

21. Parkin M. COVID-19 preprint projections. Disponible en: https://parkinm.gitlab.io/europe-pmc-reports/covid_preprint_projections [Consulta: 11 de junio de 2020].

22. SciSight. Disponible en: https://scisight.apps.allenai.org [Consulta: 11 de junio de 2020].

23. Thomasy H. In the race to crack Covid-19, scientists bypass peer review. Undark 2020. Disponible en: https://undark.org/2020/04/01/scientific-publishing-covid-19/[Consulta: 6 de junio de 2020].

24. Review Commons. Disponible en: https://www.reviewcommons.org [Consulta: 11 de junio de 2020].

25. Lakens D. Pandemic researchers: recruit your own best critics. Nature 2020; 581(7807):121. doi: 10.1038/d41586020-01392-8.

26. Chambers C. What's next for registered reports? Nature 2019; 573(7773):187-9. doi: 10.1038/d41586-019-02674-6.

27. Singh Chawla D. Opinion: it's time to lift the veil on peer review Undark 2019. Disponible en: https://undark.org/2019/06/20/lifting-the-veil-on-peer-review/ [Consulta: 5 de junio de 2020].

28. Responsible journals. Disponible en: https://www.responsiblejournals.org [Consulta: 11 de junio de 2020].

\section{Contribución de los autores}

Carolina Scasso, https://orcid.org/0000-0002-1179-4841. Diseño, análisis, redacción.

Julio Medina, https://orcid.org/0000-0001-9761-6829. Concepción, análisis y revisión crítica. 